\title{
- Performance Analysis of Multihop Wireless Links Over Generalized- $K$ Fading Channels
}

\author{
Jianfei Cao, Student Member, IEEE, Lie-Liang Yang, Senior Member, IEEE, and Zhangdui Zhong
}

\begin{abstract}
4 Abstract-The performance of multihop links is studied in 5 this contribution by both analysis and simulations when com6 municating over generalized- $K\left(K_{G}\right)$ fading channels. The per7 formance metrics considered include symbol error rate (SER), 8 outage probability, level crossing rate (LCR), and average outage 9 duration (AOD). First, the expressions for both the SER and 10 outage probability are derived by approximating the probability 11 density function (pdf) of the end-to-end signal-to-noise ratio (SNR) 12 using an equivalent end-to-end pdf. We show that this equivalent 13 end-to-end pdf is accurate for analyzing the outage probability. 14 Then, the second-order statistics of LCR and AOD of multihop 15 links are analyzed. Finally, the performance of multihop links 16 is investigated by either simulations or evaluation of the expres17 sions derived. Our performance results show that the analytical 18 expressions obtained can be well justified by the simulation results. 19 The studies show that the $\boldsymbol{K}_{\boldsymbol{G}}$ channel model and the expressions 20 derived in this paper are highly efficient for the prediction of 21 the performance metrics and statistics for the design of multihop 22 communication links.
\end{abstract}

23 Index Terms-Average outage duration (AOD), generalized- $K$ 24 distribution, level crossing rate (LCR), multihop communica25 tions, outage probability, performance analysis, symbol error 26 rate (SER).

\section{INTRODUCTION}

28 N WIRELESS communications systems, radio signals ex29 perience (large-scale) propagation path loss and shadowing, 30 as well as small-scale fading [1]. Conventionally, the effect of 31 wireless channels on system performance is investigated by 32 separately treating these phenomena, typically assuming two 33 to four orders of power decay for the propagation path loss; 34 lognormal distribution for the shadowing; and Rayleigh, Rician, 35 or Nakagami- $m$ distribution for the small-scale fading [2]. 36 To accurately predict the achievable performance, it is highly 37 desirable that a model can simultaneously cope with all the 38 aforementioned phenomena, including propagation path loss, 39 shadowing, and small-scale fading. Because of this, composite 40 channel models have been formed by combining the distrib-

Manuscript received May 25, 2011 revised November 23, 2011; accepted January 27, 2012. This work was supported in part by the Joint State Key Program of the National Natural Science Foundation of China under Grant 60830001 and in part by the Fundamental Research Funds for the Central Universities under Grant 2010JBZ008. This work was carried out in the framework of COST Action IC0905 "TERRA." This paper was presented in part at the IEEE Fall Vehicular Technology Conference, Ottawa, ON, Canada, September 3-6, 2010. The review of this paper was coordinated by Dr. C.-C. Chong.

J. Cao and Z. Zhong are with Beijing Jiaotong University, Beijing 100044, China (e-mail: cao.jianfei@hotmail.com; zhdzhong@bjtu.edu.cn).

L.-L. Yang is with the School of Electronics and Computer Science, University of Southampton, SO17 1BJ Southampton, U.K. (e-mail: 1ly@ecs.soton. ac.uk).

Digital Object Identifier 10.1109/TVT.2012.2188050 utions for small-scale fading with lognormal distribution for 41 shadowing, yielding Rayleigh-, Rician-, Nakagami-lognormal, 42 etc., composite channel models [2]. However, these compos- 43 ite channel models often result in complicated performance 44 analysis.

In recent years, the generalized- $K\left(K_{G}\right)$ channel model 46 has been proposed [3], which is formed by first approximat- 47 ing the lognormal distribution using the Gamma distribution 48 [2] and then combining it with the Nakagami- $m$ distribu- 49 tion. It has been recognized [3] that the $K_{G}$ channel model 50 can simultaneously take into account propagation path loss, 51 shadowing, and small-scale fading. It can usually cover more 52 communications scenarios encountered in real mobile wireless 53 systems, compared with the other composite channel models. 54 Furthermore, the $K_{G}$ channel model often results in closed- 55 form solutions when applied for system performance analysis 56 [4]-[6]. Therefore, in this paper, we are interested in analyzing 57 the performance of multihop links when they are operated in 58 wireless channels characterized by the $K_{G}$ channel model. The 59 symbol error rate (SER), outage probability, level crossing rate 60 (LCR), and average outage duration (AOD) of the multihop 61 links are analyzed. A range of closed-form expressions is 62 obtained. Furthermore, the performance of the multihop links is 63 investigated by both simulations and evaluation of the formulas 64 derived.

By dividing a long transmission link into multiple more 66 reliable short links supported by relays, multihop transmission 67 has the potential to improve the system's energy efficiency and 68 extend the coverage area. Recently, the performance analysis 69 of relay networks has received much attention. Specifically, in 70 [7], the general framework for studying the outage probability 71 of relay links over Nakagami- $m$ fading channels has been 72 proposed. By assuming fixed relay gain, in [8], Wu et al. 73 have investigated the performance of nonregenerative dual-hop 74 links over $K_{G}$ fading channels. The second-order statistical 75 properties, including both the LCR and AOD, of the regener- 76 ative multihop links have been analyzed over general multipath 77 fading channels [9]. Furthermore, in [10], Krantzik and Wolf 78 have studied the LCR and AOD performance of the point- 79 to-point links when communicating over the modified Suzuki 80 fading channels, which are formed by the product of two 81 independent random processes, with their envelope following 82 Rayleigh and lognormal distributions, respectively. However, 83 to the best of our knowledge, the performance of the multihop 84 links aided by regenerative relays for communications over $K_{G} 85$ fading channels has not been analyzed in the open literature. $\quad 86$

The remainder of this paper is organized as follows: 87 Section II provides a brief overview of the $K_{G}$ fading channels 88 


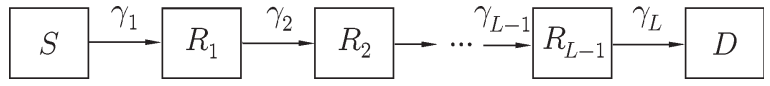

Fig. 1. Schematic of a multihop transmission link, where source $S$ sends messages to destination $D$ through $(L-1)$ relays, and the SNR of the $l$ th hop is denoted by $\gamma_{l}$ for $l=1,2, \ldots, L$.

89 and derives the equivalent probability density function (pdf) of 90 the multihop link's end-to-end signal-to-noise ratio (SNR). In 91 Section III, the average SER and outage probability are studied. 92 Section IV addresses the second-order statistics of both the $93 \mathrm{LCR}$ and AOD. Section V provides performance results, and 94 finally, in Section VI, we summarize the conclusions.

\section{II. Statistics of End-To-End Signal-to-Noise Ratio}

\section{A. System and Channel Model}

97 We consider an $L$-hop link as shown in Fig. 1, where source $98 S$ sends messages to destination $D$ via $(L-1)$ intermediate 99 relays that are expressed as $R_{1}, R_{2}, \ldots, R_{L-1}$, respectively, 100 in time-division principles. We assume that the $(L+1)$ nodes 101 are sufficiently separated, so that a node can only receive 102 signals from its adjacent nodes. The relays are regenerative 103 and are operated under the decode-and-forward cooperative 104 strategy. The SNRs of the $L$ hops are denoted by $\gamma_{1}, \gamma_{2}, \ldots, \gamma_{L}$, 105 respectively. This type of communication links exist in wireless 106 ad-hoc networks, wireless sensor networks, etc.

107 Let us assume that the envelops of the $L$ hops are expressed 108 as $Z_{1}, Z_{2}, \ldots, Z_{L}$. Then, when communicating over the $K_{G}$ 109 fading channels, these envelops obey the $K_{G}$ distribution with 110 the pdf [4]

$$
\begin{array}{r}
f_{Z_{l}}\left(z_{l}\right)=\frac{4 z_{l}^{\beta_{l}}}{\Gamma\left(m_{l}\right) \Gamma\left(k_{l}\right)}\left(\frac{m_{l} k_{l}}{\Omega_{l}}\right)^{\left(\beta_{l}+1\right) / 2} K_{\alpha_{l}}\left[2 \sqrt{\frac{m_{l} k_{l}}{\Omega_{l}}} z_{l}\right] \\
z_{l} \geq 0, l=1,2, \ldots, L
\end{array}
$$

111 where $\alpha_{l}=k_{l}-m_{l}, \beta_{l}=k_{l}+m_{l}-1, k_{l}$ and $m_{l}$ are the dis112 tribution shaping parameters accounting for the shadowing and 113 Nakagami- $m$ (small-scale) fading associated with the $l$ th hop, $114 K_{\alpha_{l}}(\cdot)$ is the second kind modified Bessel function of order $115 \alpha_{l}$, and $\Gamma(\cdot)$ denotes the Gamma function. In (1), $\Omega_{l}=E\left\{Z_{l}^{2}\right\}$, 116 where $E\{\cdot\}$ is the expectation operation, represents the local 117 mean determined by propagation path loss [3]. In practice, $\Omega_{l}$ 118 can be obtained by the relatively long-time average over various 119 locations within a certain area to remove the effects of both 120 shadowing and small-scale fading. Specifically, when giving a 121 transmission distance $d_{l}$ and a propagation path-loss exponent $122 \eta$, we then have $\Omega_{l}=d_{l}^{-\eta}$. Therefore, by controlling the values 123 of the shaping parameters $k_{l}$ and $m_{l}$, as well as the value of $124 \Omega_{l}$, (1) can be used to approximate the channel distribution 125 in numerous communication environments. Multipath fading, 126 shadowing, the composite of shadowing and multipath fading, 127 additive white Gaussian noise (AWGN) channels, etc., can all 128 be modeled by the distribution of (1) by appropriately setting 129 the parameters of $k_{l}, m_{l}$, and $\Omega_{l}$ [6].

130 To facilitate the performance analysis, usually, the pdf of the 131 instantaneous SNR per hop of $\gamma_{l}=Z_{l}^{2} E / N_{0}$ is required, where $132 E$ denotes the common energy per symbol, and $N_{0}$ is the single- sided power spectral density of AWGN. This pdf can be readily 133 derived from (1) and can be expressed as

$$
f_{\gamma_{l}}(\gamma)=\frac{2 \Psi_{l}^{\left(\beta_{l}+1\right) / 2} \gamma^{\left(\beta_{l}-1\right) / 2}}{\Gamma\left(m_{l}\right) \Gamma\left(k_{l}\right)} K_{\alpha_{l}}\left[2 \sqrt{\Psi_{l} \gamma}\right], \gamma \geq 0
$$

where $\Psi_{l}=m_{l} k_{l} / \bar{\gamma}_{l}$, and $\bar{\gamma}_{l}=\Omega_{l} E / N_{0}$.

As shown in Fig. 1, the relay link has the property of re- 136 generative transmission. Hence, if any of the first $(L-1)$ hops 137 suffers from severe fading, resulting in an outage event that its 138 SNR falls below a preset threshold $\gamma_{\text {th }}$, the whole transmission 139 from source $S$ to destination $D$ is apparently failed. Otherwise, 140 if the last relay $R_{L-1}$ can successfully decode the messages 141 transmitted by source $S$, then the statistical property of the SNR 142 observed at destination $D$ is mainly determined by that of the 143 last hop's SNR. Therefore, inspired by the approaches proposed 144 in [11], the cascaded multihop transmissions from source $S 145$ to destination $D$, as shown in Fig. 1, can be reduced to an 146 equivalent point-to-point link to simplify the analysis. With the 147 aid of this equivalency, the pdf of the end-to-end SNR of Fig. 1148 can be represented as [11]

$$
f(\gamma)=A \delta(\gamma)+(1-A) f_{\gamma_{L}}(\gamma)
$$

where $A$ is the probability that outage occurs with the first 150 $(L-1)$ hops, whereas $f_{\gamma_{L}}(\gamma)$ denotes the pdf of the last hop's 151 SNR. Explicitly, the equivalent pdf of the end-to-end SNR is 152 constituted by two components, i.e., the discrete component of 153 $P(\gamma=0)=A$ and the continuous $K_{G}$ pdf scaled by a factor 154 $(1-A)$.

We assume that the channels of the $L$ hops are independent, 156 which is reasonable, provided that any two of the $(L+1)$ nodes 157 in Fig. 1 are separated by a sufficient distance, such as by a few 158 wavelengths. In this case, the SNRs $\gamma_{l}, l=1, \ldots, L$ are also 159 mutually independent and have the pdfs $f_{\gamma_{l}}(\gamma), l=1, \ldots, L, 160$ as shown in (2). Note that, since the SNRs $\gamma_{l}, l=1, \ldots, L$ may 161 be associated with different values for parameters $k_{l}, m_{l}$, and 162 $\Omega_{l}$, the pdfs $f_{\gamma_{l}}(\gamma), l=1, \ldots, L$ may be different. Under the 163 aforementioned assumption, when given a preset threshold $\gamma_{\text {th }}, 164$ the probability $A$ in (3) can be derived as

$$
\begin{aligned}
A & =1-\operatorname{Pr}\left\{\gamma_{1}>\gamma_{\mathrm{th}}, \gamma_{2}>\gamma_{\mathrm{th}}, \ldots, \gamma_{L-1}>\gamma_{\mathrm{th}}\right\} \\
& =1-\prod_{l=1}^{L-1} \operatorname{Pr}\left\{\gamma_{l}>\gamma_{\mathrm{th}}\right\} \\
& =1-\prod_{l=1}^{L-1}\left[1-F_{\gamma_{l}}\left(\gamma_{\mathrm{th}}\right)\right]
\end{aligned}
$$

where $F_{\gamma_{l}}(\cdot)$ represents the cumulative distribution function 166 (cdf) of $\gamma_{l}$, which, for noninteger parameter $\alpha_{l}$, is given by [6] 167

$$
\begin{aligned}
F_{\gamma_{l}}(\gamma)=\pi & \csc \left(\pi \alpha_{l}\right) \\
\times & \times\left[\frac{\left(\Psi_{l} \gamma\right)^{m_{l}}{ }_{1} F_{2}\left(m_{l} ; 1-\alpha_{l}, 1+m_{l} ; \Psi_{l} \gamma\right)}{\Gamma\left(k_{l}\right) \Gamma\left(1-\alpha_{l}\right) \Gamma\left(1+m_{l}\right)}\right. \\
& \left.\quad-\frac{\left(\Psi_{l} \gamma\right)^{k_{l}}{ }_{1} F_{2}\left(k_{l} ; 1+\alpha_{l}, 1+k_{l} ; \Psi_{l} \gamma\right)}{\Gamma\left(m_{l}\right) \Gamma\left(1+\alpha_{l}\right) \Gamma\left(1+k_{l}\right)}\right], \gamma \geq 0
\end{aligned}
$$


168 where ${ }_{p} F_{q}(a ; b, c ; z)$ is the generalized hypergeometric func169 tion with integer parameters $p$ and $q$, as defined in [12]. When 170 parameters $m_{l}$ and $k_{l}$ are integers, then the cdf of $\gamma_{l}$ can be 171 expressed as [13]

$$
\begin{aligned}
F_{\gamma_{l}}(\gamma)=1- & \frac{2\left(\Psi_{l} \gamma\right)^{k_{l} / 2}}{\Gamma\left(k_{l}\right)} \\
& \times \sum_{q=0}^{m_{l}-1} \frac{1}{q !}\left(\Psi_{l} \gamma\right)^{q / 2} K_{k_{l}-q}\left[2 \sqrt{\Psi_{l} \gamma}\right], \gamma>0 .
\end{aligned}
$$

\section{B. Moments of End-to-End SNR}

173 With the equivalent pdf, as shown in (3), the $n$th moment of 174 the end-to-end SNR $\mu_{\gamma}(n)$ can be evaluated by the formula

$$
\mu_{\gamma}(n)=\int_{0}^{\infty} \gamma^{n} f(\gamma) d \gamma
$$

175 Upon substituting (3) into (7) and using [12, eq. (6.561/16)], we 176 obtain

$$
\mu_{\gamma}(n)=(1-A) \frac{\Gamma\left(k_{L}+n\right) \Gamma\left(m_{L}+n\right)}{\Gamma\left(k_{L}\right) \Gamma\left(m_{L}\right) \Psi_{L}^{n}}
$$

177 Furthermore, with the aid of (8), the amount of fading (AoF) 178 often used to measure the severity of fading can be computed 179 by the formula [2]

$$
\mathrm{AoF}=\frac{\mu_{\gamma}(2)}{\mu_{\gamma}^{2}(1)}-1
$$

180 Let us now analyze the SER and outage probability of the 181 general multihop links, as shown in Fig. 1, when they are 182 operated in $K_{G}$ fading channels.

\section{III. ANALYSIS OF SYMBOL ERROR RATE 184 AND OUTAGE PROBABILITY}

185 In this section, we adopt the moment generation function 186 (MGF) approach [2] to analyze the SER and outage perfor187 mance of the multihop links over $K_{G}$ fading channels. The $188 \mathrm{MGF} M_{\gamma}(s)$ of the end-to-end SNR can be expressed as

$$
M_{\gamma}(s)=\int_{0}^{\infty} \exp (-\gamma s) f(\gamma) d \gamma .
$$

189 By substituting (3) into (10) and using [12, eq. (6.643/3)], it 190 can be shown that the MGF of the end-to-end SNR can be ex191 pressed as

$$
\begin{aligned}
M_{\gamma}(s)=A+(1-A)\left(\frac{\Psi_{L}}{s}\right)^{\frac{\beta_{L}}{2}} & \exp \left(\frac{\Psi_{L}}{2 s}\right) \\
& \times W_{-\frac{\beta_{L}}{2}, \frac{\alpha_{L}}{2}}\left(\frac{\Psi_{L}}{s}\right)
\end{aligned}
$$

192 where $W_{v, \mu}(z)$ is the Whittaker's function, as defined in [12].

\section{A. Average SER}

For many coherent demodulation schemes, such as 194 $M$-ary amplitude shift keying, binary phase-shift keying 195 (BPSK), binary frequency-shift keying (BFSK), etc., the SER 196 $P_{e}(\gamma)$ conditioned on the SNR $\gamma$ can be expressed in the form 197 as [2], [14]

$$
P_{e}(\gamma)=a \int_{0}^{\frac{\pi}{2}} \exp \left(-\frac{g \gamma}{\sin ^{2} \theta}\right) d \theta
$$

where parameters $a$ and $g$ are determined by the specific 199 modulation scheme employed. For example, for BPSK, we 200 have $a=1 / \pi$ and $g=1$. The average SER of the multihop 201 links using a specific modulation scheme can be evaluated by 202 integrating $P_{e}(\gamma)$ with respect to the pdf of $\gamma$, i.e.,

$$
\bar{P}_{e}=\int_{0}^{\infty} P_{e}(\gamma) f(\gamma) d \gamma
$$

Upon substituting (3) and (12) into the aforementioned equation 204 and exchanging the order of integration, we obtain

$$
\begin{aligned}
\bar{P}_{e} & =a \int_{0}^{\frac{\pi}{2}} \int_{0}^{\infty} \exp \left(-\frac{g \gamma}{\sin ^{2} \theta}\right) f(\gamma) d \gamma d \theta \\
& =a \int_{0}^{\frac{\pi}{2}} M_{\gamma}\left(\frac{g}{\sin ^{2} \theta}\right) d \theta
\end{aligned}
$$

which relates the average SER to the MGF of (11). Therefore, 206 when applying (11) into (14), the average SER can be ex- 207 pressed as

$$
\begin{aligned}
\vec{P}_{e}= & \frac{\pi a A}{2}+a(1-A)\left(\frac{\Psi_{L}}{g}\right)^{\frac{\beta_{L}}{2}} \int_{0}^{\frac{\pi}{2}}(\sin \theta)^{\beta_{L}} \\
& \times \exp \left(\frac{\Psi_{L} \sin ^{2} \theta}{2 g}\right) W_{\frac{-\beta_{L}}{2}, \frac{\alpha_{L}}{2}}\left(\frac{\Psi_{L} \sin ^{2} \theta}{g}\right) d \theta .
\end{aligned}
$$

To simplify (15), we first make use of the formula [15, 209 eq. (07.45.26.0005.01)]

$$
\begin{aligned}
e^{z / 2} W_{v, \mu}(z)=\frac{1}{\Gamma(1 / 2-\mu-v) \Gamma(\mu-v+1 / 2)} \\
\times G_{1,2}^{2,1}\left(\begin{array}{c|c}
z+1 \\
\mu+1 / 2,1 / 2-\mu
\end{array}\right)
\end{aligned}
$$

where $G(\cdot)$ is the Meijer's $G$-function, as defined 211 in [12, eq. (9.301)]. Then, we make the variable transform of 212 
$213 t=\sin ^{2} \theta$. Finally, after some simplifications with the aid of 214 [12, eq. (7.811.2)], we obtain the average SER

$$
\begin{aligned}
\bar{P}_{e}= & \frac{\pi a A}{2}+\frac{a(1-A) \Gamma\left(\frac{1}{2}\right)}{2 \Gamma\left(\frac{1-\alpha_{L}+\beta_{L}}{2}\right) \Gamma\left(\frac{1+\alpha_{L}+\beta_{L}}{2}\right)}\left(\frac{\Psi_{L}}{g}\right)^{\frac{\beta_{L}}{2}} \\
& \times G_{2,3}^{2,2}\left(\frac{\Psi_{L}}{g} \mid \begin{array}{c}
\left(1-\beta_{L}\right) / 2,\left(2-\beta_{L}\right) / 2 \\
\left(1+\alpha_{L}\right) / 2,\left(1-\alpha_{L}\right) / 2,-\beta_{L} / 2
\end{array}\right) .
\end{aligned}
$$

215 When noncoherent demodulation, such as BFSK, binary 216 differential phase-shift keying, etc., employing square-law de217 tection is considered, the conditional SER can be expressed 218 as $P_{e, n o n}(\gamma)=C \exp (-D \gamma)$, where $C$ and $D$ are constants 219 determined by the corresponding noncoherent demodulation 220 scheme employed. Therefore, by averaging $P_{e, n o n}(\gamma)$ using 221 the pdf of (3) and with the aid of [12, eq. (6.643/3)], we can 222 obtain the average SER of the multihop links over $K_{G}$ fading 223 channels, which is

$$
\begin{aligned}
\bar{P}_{e, n o n}=C A+C(1 & -A)\left(\frac{\Psi_{L}}{D}\right)^{\frac{\beta_{L}}{2}} \\
& \times \exp \left(\frac{\Psi_{L}}{2 D}\right) W_{-\frac{\beta_{L}}{2}, \frac{\alpha_{L}}{2}}\left(\frac{\Psi_{L}}{D}\right) .
\end{aligned}
$$

224 Let us now analyze the outage probability.

\section{B. Outage Probability}

226 The outage event occurs provided that there is at least a hop 227 having its SNR lower than a threshold $\gamma_{\mathrm{th}}$. Using the equivalent $228 \mathrm{pdf}$ of (3), we have the outage probability

$$
P_{\text {out }}=\int_{0}^{\gamma_{\text {th }}} f(\gamma) d \gamma
$$

229 By substituting (3) into (19), it can be readily shown that the 230 outage probability can be represented as

$$
P_{\text {out }}=A+(1-A) F_{\gamma_{L}}\left(\gamma_{\mathrm{th}}\right) \text {. }
$$

231 The outage probability can also be directly derived from the 232 multihop links of Fig. 1, which can be expressed as

$$
\begin{aligned}
P_{\mathrm{out}} & =1-P\left[\gamma_{1}>\gamma_{\mathrm{th}}, \gamma_{2}>\gamma_{\mathrm{th}}, \ldots, \gamma_{L}>\gamma_{\mathrm{th}}\right] \\
& =1-\prod_{l=1}^{L} \operatorname{Pr}\left\{\gamma_{l}>\gamma_{\mathrm{th}}\right\} \\
& =1-\prod_{l=1}^{L}\left[1-F_{\gamma_{l}}\left(\gamma_{\mathrm{th}}\right)\right] .
\end{aligned}
$$

233 Let $\bar{A}=\prod_{l=1}^{L-1}\left[1-F_{\gamma_{l}}\left(\gamma_{\mathrm{th}}\right)\right]$ in the aforementioned equation. 234 According to (4), explicitly, we have $A+\bar{A}=1$. Upon apply235 ing them into (21), we can readily prove that (21) is the same 236 as (20). This implies that the equivalent model associated with 237 the pdf of (3) is accurate in terms of the outage probability.

\section{LeVel CRossing Rate AND Average Outage Duration}

In addition to the performance measurements based on the 240 average SER and outage probability, in wireless communica- 241 tions, the LCR and AOD are also very important for system 242 design and optimization. For example, both the LCR and AOD 243 have a strong impact on the selection of packet length, channel- 244 coding schemes, length of interleaver, etc. The reader who 245 is interested in more details about the impact of LCR and 246 AOD on system design and optimization is referred to [9] and 247 [16]-[18], as well as the references therein. Therefore, in this 248 section, we study the LCR and AOD of the multihop links. To 249 the best of the authors' knowledge, the second-order statistics 250 of the LCR and AOD of multihop links operated over $K_{G} 251$ fading channels have not been studied in literature. Note that, 252 the LCR is used to quantify how often the fading process 253 crosses a preset threshold, usually in the positive-going direc- 254 tion, whereas the AOD is defined as the average period of time 255 during which the channel quality, such as SNR, is below a 256 predefined threshold [9], [16]-[18].

257

Here, we first derive the LCR and AOD in the context of the 258 $l$ th hop when it is operated over $K_{G}$ fading channels. Then, the 259 results are extended to the multihop paradigms with the aid of 260 the approaches proposed in [9]. For the sake of simplicity, the 261 subscript/superscript $l$ is dropped during our analysis of the $l$ th 262 hop, yielding no confusion.

According to [18], the AOD $T\left(z_{\mathrm{th}}\right)$ in seconds can be 264 approximately expressed as

$$
T\left(z_{\mathrm{th}}\right) \approx \frac{P_{\mathrm{out}}}{N\left(z_{\mathrm{th}}\right)}
$$

where $P_{\text {out }}$ is the outage probability, which has been derived 266 in Section III-B, and $N\left(z_{\text {th }}\right)$ represents the LCR for a given 267 threshold $z_{\mathrm{th}}$. Therefore, to derive $T\left(z_{\mathrm{th}}\right)$ of the AOD, we first 268 need to obtain $N\left(z_{\mathrm{th}}\right)$ of the LCR, which can be evaluated by 269 the integration [19]

$$
N\left(z_{\mathrm{th}}\right)=\int_{0}^{\infty} \dot{z} f_{Z \dot{Z}}\left(z_{\mathrm{th}}, \dot{z}\right) d \dot{z}
$$

where $f_{Z \dot{Z}}(z, \dot{z})$ represents the joint pdf of the random 271 processes $Z(t)$ and its time derivative $\dot{Z}=d Z(t) / d t$ at 272 time $t$.

To derive the joint pdf of $f_{Z \dot{Z}}(z, \dot{z})$, we can commence from 274 the composite structure of the $K_{G}$ distribution [5]

$$
f_{Z}(z)=\int_{0}^{\infty} f_{Z \mid Y}(z \mid y) f_{Y}(y) d y
$$

where $f_{Z}(z)$ is the $K_{G}$ distribution in the form of (1), 276 $f_{Z \mid Y}(z \mid y)$ denotes the pdf of the Nakagami- $m$ distribution 277 conditioned on the local mean $Y=y$, and $f_{Y}(y)$ represents the 278 Gamma pdf approximating the distribution of the local mean. 279 
280 In detail, the conditional Nakagami- $m$ and Gamma pdfs for 281 deriving the $K_{G}$ distribution shown in (1) are given by

$$
\begin{aligned}
f_{Z \mid Y}(z \mid y) & =\frac{2 m^{m}}{\Gamma(m) y^{m}} z^{2 m-1} \exp \left(-\frac{m z^{2}}{y}\right) \\
f_{Y}(y) & =\frac{k^{k}}{\Gamma(k) \Omega^{k}} y^{k-1} \exp \left(-\frac{k y}{\Omega}\right) .
\end{aligned}
$$

282 Let us use the variable transform of $S=Z / Y$ in (25). It can 283 be shown that, for a given value of $Y$, variable $S$ also obeys 284 the Nakagami- $m$ distribution, which is related to the original 285 Nakagami- $m$ pdf through the relationship of

$$
f_{Z \mid Y}(z \mid y)=\frac{1}{y} f_{S}\left(\frac{z}{y}\right)
$$

286 where $f_{S}(\cdot)$ represents the pdf of variable $S$. Upon applying the 287 preceding relationship into (24), we obtain

$$
f_{Z}(z)=\int_{0}^{\infty} \frac{1}{y} f_{S}\left(\frac{z}{y}\right) f_{Y}(y) d y .
$$

288 Finally, according to [10], the joint pdf of $Z(t)$ and $\dot{Z}(t)$ can be 289 expressed in the integral form as

$$
f_{Z \dot{Z}}(z, \dot{z})=\int_{0}^{\infty} \frac{1}{y^{2}} \int_{-\infty}^{\infty} f_{S \dot{S}}\left(\frac{z}{y},\left(\frac{\dot{z}}{y}\right)\right) f_{Y \dot{Y}}(y, \dot{y}) d y d \dot{y}
$$

290 where $f_{S \dot{S}}(\cdot, \cdot)$ represents the joint pdf of the random process $291 S(t)$ and its derivative $\dot{S}(t)$, and $f_{Y \dot{Y}}(\cdot, \cdot)$ represents the joint 292 pdf of the random process $Y(t)$ and its derivative $\dot{Y}(t)$.

293 Since the Nakagami- $m$ distributed random process and 294 its time derivative are mutually independent [20], we have $295 f_{S \dot{S}}(\cdot, \cdot)=f_{S}(\cdot) f_{\dot{S}}(\cdot)$. Furthermore, according to [20], the 296 samples from the time derivative of a Nakagami- $m$ distributed 297 random process obeys the well-known Gaussian distribution 298 with zero mean and a variance of $\sigma_{S}^{2}=f_{m}^{2} \pi^{2} / y m$, where $f_{m}=$ $299 f_{m}^{(l)}$ denotes the maximum Doppler frequency shift in the con300 text of the $l$ th hop. Hence, the joint pdf $f_{S \dot{S}}(z / y,(z / y))$ seen 301 in (29) can be obtained as the product of the Nakagami- $m$ dis302 tribution of $f_{S}(z / y)$ and the Gaussian distribution $f_{\dot{S}}((z \dot{/} y))$, 303 which is expressed as

$$
\begin{aligned}
f_{S \dot{S}}\left(\frac{z}{y},\left(\frac{\dot{z}}{y}\right)\right) & =\frac{2 m^{m}}{\Gamma(m) y^{m-1}} z^{2 m-1} \exp \left(-\frac{m z^{2}}{y}\right) \\
& \times \frac{1}{\sqrt{2 \pi} \sigma_{S}} \exp \left(-\left(\frac{\dot{z}}{y}-\frac{z \dot{y}}{y^{2}}\right)^{2} / 2 \sigma_{S}^{2}\right)
\end{aligned}
$$

304 where $(z \dot{\gamma} y)=\dot{z} / y-z \dot{y} / y^{2}$ has been applied.

305 Unfortunately, a Gamma-distributed random process and its 306 time derivative are not independent. Hence, we cannot derive 307 the pdf $f_{Y \dot{Y}}(y, \dot{y})$ in the same way as for the Nakagami- $m$ dis308 tributed random process. However, the joint pdf of $f_{Y \dot{Y}}(y, \dot{y})$ 309 can be obtained from [21], which studies the joint pdf between 310 the random process obeying the generalized Gamma distribu311 tion and its time derivative. It can be shown that the generalized
Gamma distribution reduces to the Gamma distribution of (26) 312 when the parameter $\beta$ in [21, eq. (4)] equals one. Therefore, by 313 setting $\beta=1$ in [21, eq. (15)], we obtain the joint pdf of the 314 Gamma-distributed random process and its time derivative as 315

$$
\begin{aligned}
f_{Y \dot{Y}}(y, \dot{y})=\frac{k^{k+1 / 2}}{2 \Gamma(k) \Omega^{k+1 / 2}} \sqrt{-\rho^{\prime \prime}(0) \pi} & y^{k-\frac{3}{2}} \\
& \times \exp \left(\frac{k \dot{y}^{2}}{4 \Omega \rho^{\prime \prime}(0) y}-\frac{k y}{\Omega}\right)
\end{aligned}
$$

where $\rho(\tau)$ is the normalized correlation function of the 316 Gamma-distributed random process, and $\rho^{\prime \prime}(0)$ is its second- 317 order derivative at $\tau=0$. For example, for land mobile com- 318 munication systems, usually, $\rho(\tau)=J_{0}\left(2 \pi f_{m} \tau\right)$, where $J_{0}(x) 319$ is the zeroth-order Bessel function of the first kind. Correspond- 320 ingly, we have $\rho^{\prime \prime}(0)=-2 \pi^{2} f_{m}^{2}$.

321

Finally, when substituting the joint pdfs of (30) and (31) into 322 (29) and then using [12, eq. (3.321.4)] to simplify it, we can 323 obtain the joint pdf of the $K_{G}$ distributed random process $Z(t) 324$ and its time derivative $\dot{Z}(t)$ as

325

$$
\begin{aligned}
f_{Z, \dot{z}}(z, \dot{z}) & =\frac{2 \sqrt{a b} m^{m} k^{k} z^{2 m-1}}{\sqrt{\pi} \Gamma(m) \Gamma(k) \Omega^{k}} \int_{0}^{\infty} \frac{y^{\alpha}}{\sqrt{a y^{3}+b z^{2} y}} \\
& \times \exp \left(-\frac{k}{\Omega} y-\frac{b \dot{z}+m z^{2}}{y}+\frac{b^{2} z^{2} z^{2}}{a y^{3}+b z^{2} y}\right) d y
\end{aligned}
$$

where, by definition, $a=-k / 4 \Omega \rho^{\prime \prime}(0)$, and $b=m / 2 \pi^{2} f_{m}^{2} . \quad 326$

Furthermore, let $z=z_{\text {th }}$ in (32). Then, when substituting 327 (32) into (23) and completing the integration with respect to 328 $\dot{z}$, we can obtain the LCR for a single-hop link as

$$
\begin{aligned}
N\left(z_{\mathrm{th}}\right)=\frac{m^{m} k^{k} z_{\mathrm{th}}^{2 m-1}}{\sqrt{a b \pi} \Gamma(m) \Gamma(k) \Omega^{k}} \int_{0}^{\infty} y^{\alpha-\frac{3}{2}} \\
\quad \times \sqrt{a y^{2}+b z_{\mathrm{th}}^{2}} \exp \left(-\frac{k}{\Omega} y-\frac{m z_{\mathrm{th}}^{2}}{y}\right) d y
\end{aligned}
$$

which contains just one integration that can be easily eval- 330 uated using existing software packages, such as MATLAB, 331 IT ++ , etc.

When the multihop links are considered, according to [9], the 333 end-to-end LCR over the $K_{G}$ channels can be evaluated by the 334 formula

$$
N_{T}\left(z_{\mathrm{th}}\right)=\sum_{n=1}^{L} N_{n}\left(z_{\mathrm{th}}\right) \prod_{\substack{l=1 \\ l \neq n}}^{L}\left[1-F_{Z_{l}}\left(z_{\mathrm{th}}\right)\right]
$$

where $N_{n}(\cdot)$ denotes the $n$th hop's LCR, which is given by (33) 336 with parameters $m, k, \Omega$, and $f_{m}$ replaced by $m_{n}, k_{n}, \Omega_{n}$, and 337 $f_{m}^{(n)}$, respectively. In (34), $F_{Z_{l}}(\cdot)$ denotes the cdf of $Z_{l}$ of the 338 $l$ th hop's envelope, which can be derived from (5) and (6) based 339 on the variable transform of $\gamma_{l}=\bar{\gamma}_{l} Z_{l}^{2} / \Omega_{l}$. From this variable 340 
341 transform, it can be shown that $F_{Z_{l}}(z)=F_{\gamma_{l}}\left(\bar{\gamma}_{l} z^{2} / \Omega_{l}\right)$. Hence, 342 for noninteger parameter $\alpha_{l}$, from (5), we obtain that

$$
\begin{aligned}
& F_{Z_{l}}(z) \\
& =\pi \csc \left(\pi \alpha_{l}\right) \\
& \times\left[\left(\frac{m_{l} k_{l}}{\Omega_{l}} z^{2}\right)^{m_{l}} \frac{{ }_{1} F_{2}\left(m_{l} ; 1-\alpha_{l}, 1+m_{l} ; m_{l} k_{l} z^{2} / \Omega_{l}\right)}{\Gamma\left(k_{l}\right) \Gamma\left(1-\alpha_{l}\right) \Gamma\left(1+m_{l}\right)}\right. \\
& \left.-\left(\frac{m_{l} k_{l}}{\Omega_{l}} z^{2}\right)^{k_{l}} \frac{{ }_{1} F_{2}\left(k_{l} ; 1+\alpha_{l}, 1+k_{l} ; m_{l} k_{l} z^{2} / \Omega_{l}\right)}{\Gamma\left(m_{l}\right) \Gamma\left(1+\alpha_{l}\right) \Gamma\left(1+k_{l}\right)}\right] .
\end{aligned}
$$

343 By contrast, when $m_{l}$ and $k_{l}$ are integers, we can have from 344 (6) that

$$
\begin{aligned}
F_{Z_{l}}(z)=1-\frac{2}{\Gamma\left(k_{l}\right)}\left(\frac{m_{l} k_{l}}{\Omega_{l}} z^{2}\right)^{k_{l} / 2} \sum_{q=0}^{m_{l}-1} \frac{1}{q !}\left(\frac{m_{l} k_{l}}{\Omega_{l}} z^{2}\right)^{q / 2} \\
\times K_{k_{l}-q}\left[2 \sqrt{\left.\frac{m_{l} k_{l}}{\Omega_{l}} z\right] .}\right.
\end{aligned}
$$

345 Finally, the AOD of multihop links communicating over $K_{G}$ 346 channels can be obtained by substituting $P_{\text {out }}$ of (20) and $347 N_{T}\left(z_{\text {th }}\right)$ of (34) into (22).

348 Let us now provide the simulation and numerical results 349 to characterize the performance of multihop links in the next 350 section.

\section{Performance Results}

352 In this section, the SER, outage probability, AoF, LCR, and 353 AOD performance of the multihop links communicating over $354 K_{G}$ fading channels are investigated by both simulation and 355 numerical approaches. In our investigations, we assumed that 356 the channels of the $L$ hops were independent and identically 357 distributed (i.i.d.) $K_{G}$ fading channels. The impact of propa358 gation path loss, shadowing, and Nakagami- $m$ fading on the 359 achievable performance was all considered. For convenience, 360 the intermediate relays were assumed to be equally located on 361 a line connecting source $S$ and destination $D$, making all the $362 L$ hops the same distance. We assumed that the maximum dis363 tance from source $S$ to destination $D$ was $L_{\max }=5$, after being 364 normalized by the distance of one hop. Hence, when given $L \leq$ $365 L_{\max }$ number of hops, the parameter $\Omega_{l}$ in (1), which accounts 366 for the propagation path loss of the $l$ th hop, was $\Omega_{l}=\Omega=$ $367\left(L / L_{\max }\right)^{\eta}$, where $\eta$ represents the global propagation path368 loss exponent, which was set to $\eta=3$ in our simulation and 369 numerical examples. Furthermore, for the sake of fairness of 370 comparison, the transmission power was assumed to be evenly 371 allocated to the $L$ transmitters, making $\bar{\gamma}_{l}=\bar{\gamma}=\Omega \times \mathrm{SNR} / L$, 372 where SNR is the total SNR without propagation path loss, 373 which was used for depicting the figures. Additionally, when 374 concerning the outage probability, the threshold was set accord375 ing to $\log _{2}\left(1+\gamma_{\mathrm{th}}\right) / L=R$, with the spectrum efficiency $R$ 376 being set to $0.3 \mathrm{bit} / \mathrm{s} / \mathrm{Hz}$.

377 Figs. 2-4 show the average error performance of the three378 hop $(L=3)$ links employing coherent BPSK (see Fig. 2),

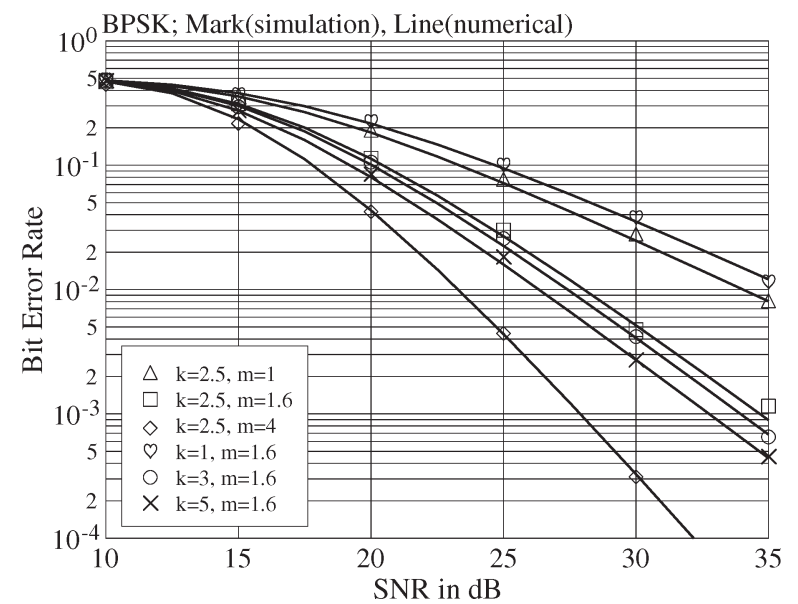

Fig. 2. BER performance of three-hop links using BPSK modulation when communicating over $K_{G}$ fading channels with $\Omega_{l}=\Omega=(3 / 5)^{3}$ and other various shaping parameters.

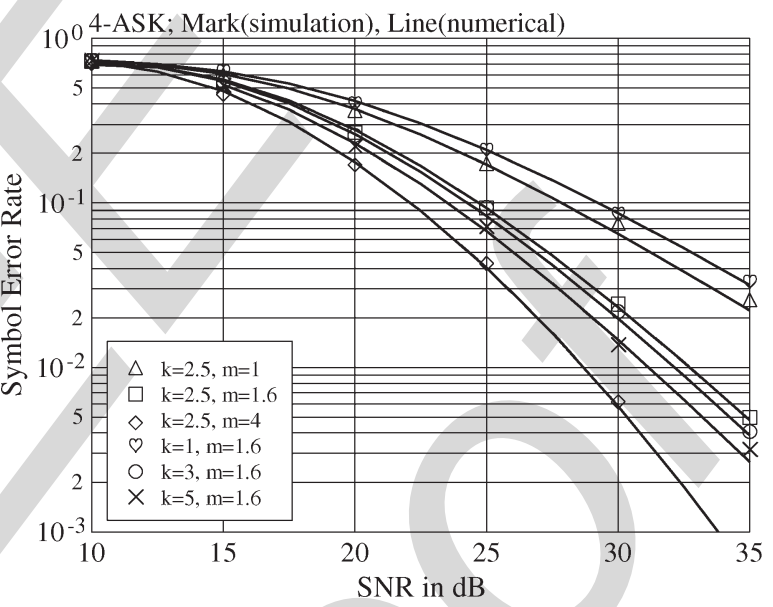

Fig. 3. SER performance of three-hop links using 4ASK modulation when communicating over $K_{G}$ fading channels with $\Omega_{l}=\Omega=(3 / 5)^{3}$ and other various shaping parameters.

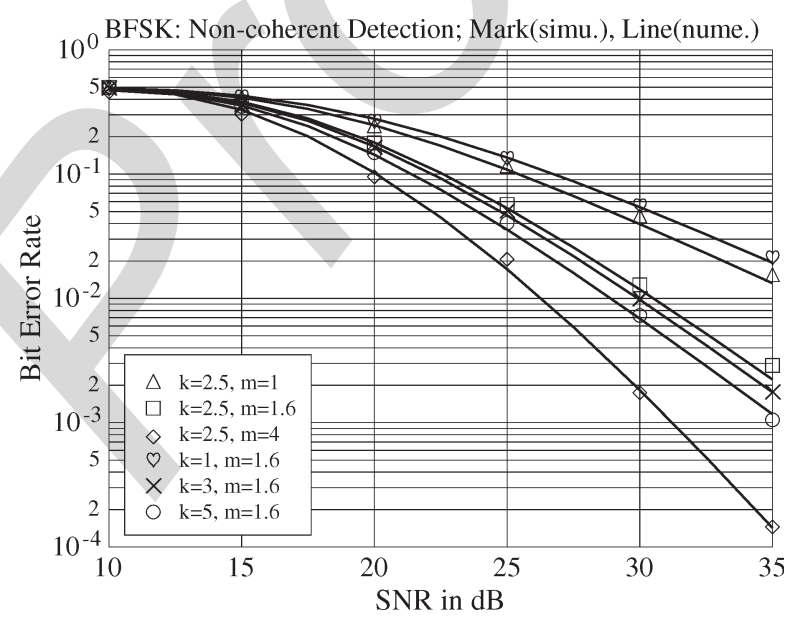

Fig. 4. BER performance of three-hop links using noncoherent BFSK modulation when communicating over $K_{G}$ fading channels with $\Omega_{l}=\Omega=(3 / 5)^{3}$ and other various shaping parameters.

coherent 4ASK (see Fig. 3), or noncoherent BFSK (see Fig. 4) 379 modulation, when communicating over the $K_{G}$ fading chan- 380 nels with various values for the shaping parameters $k$ and $m, 381$ whereas $\Omega_{l}=\Omega=(3 / 5)^{3}$. From the results of Figs. 2-4, we 382 


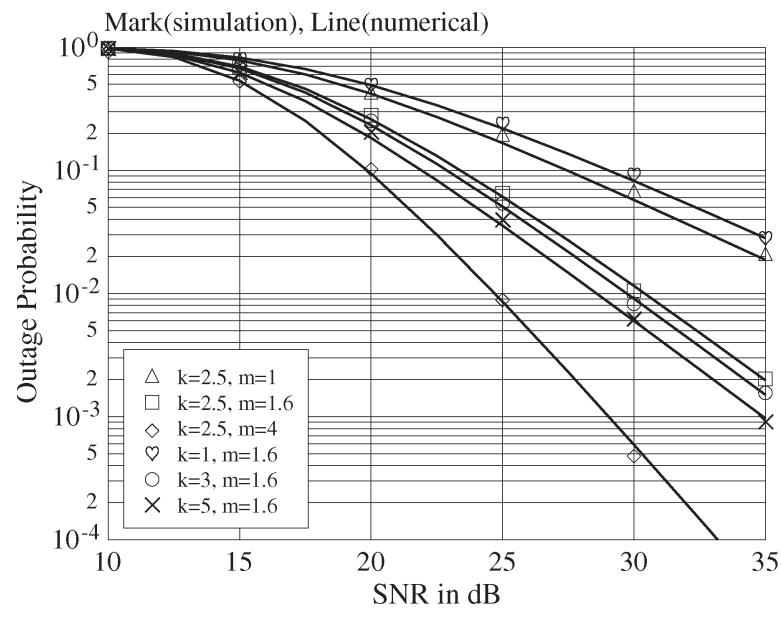

Fig. 5. Outage probability of three-hop links when communicating over $K_{G}$ fading channels with $\Omega_{l}=\Omega=(3 / 5)^{3}$ and other various shaping parameters.

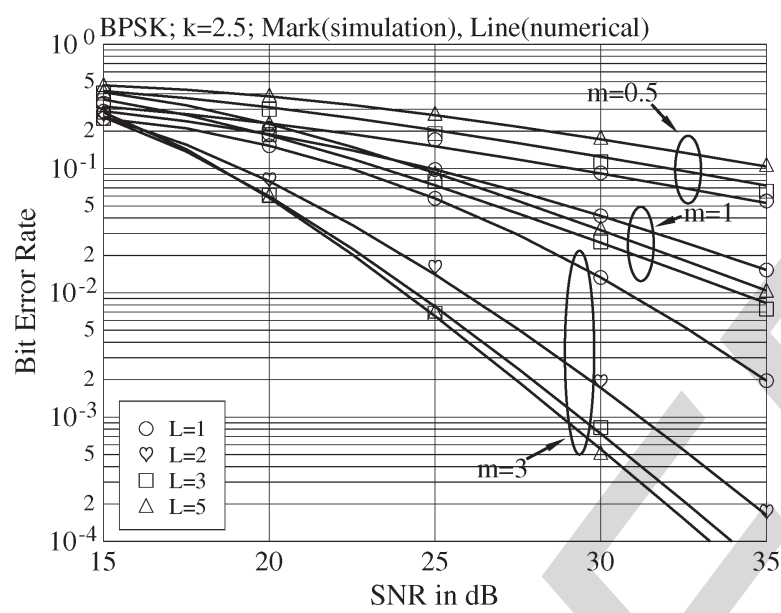

Fig. 6. BER performance of one-, two-, three-, or five-hop links using BPSK modulation when communicating over $K_{G}$ fading channels with $\Omega_{l}=\Omega=$ $(L / 5)^{3}$

383 can first observe that the numerical results agree well with the 384 simulation results. Therefore, the equivalent point-to-point link 385 with the pdf given by (3) is an effective model for studying the 386 error performance of a corresponding multihop link. Second, 387 given a fixed value of $m=1.6$ corresponding to a specific 388 small-scale fading, the BER performance of the multihop links 389 improves as the value of $k$ increases, implying that the chan390 nel increasingly shadows less. Similarly, for a given value of $391 k=2.5$, the BER performance improves as the value of $392 \mathrm{~m}$ increases, corresponding to the small-scale fading becoming 393 less severe.

394 Fig. 5 shows the exact outage probability of the three395 hop $(L=3)$ links, when communicating over the $K_{G}$ fading 396 channels with $\Omega=(3 / 5)^{3}$ and various values for the other 397 shaping parameters $k$ and $m$. Again, when the channel quality 398 improves, i.e., when the value of $k$ and/or $m$ increases, the 399 outage probability at a given SNR decreases. However, it seems 400 that the outage performance is sensitive more to the small-scale 401 fading determined by the value of $m$ than to the shadowing 402 determined by the value of $k$.

403 Figs. 6 and 7 investigate the effect of the number of hops 404 on the achievable BER (see Fig. 6) or outage (see Fig. 7) per-

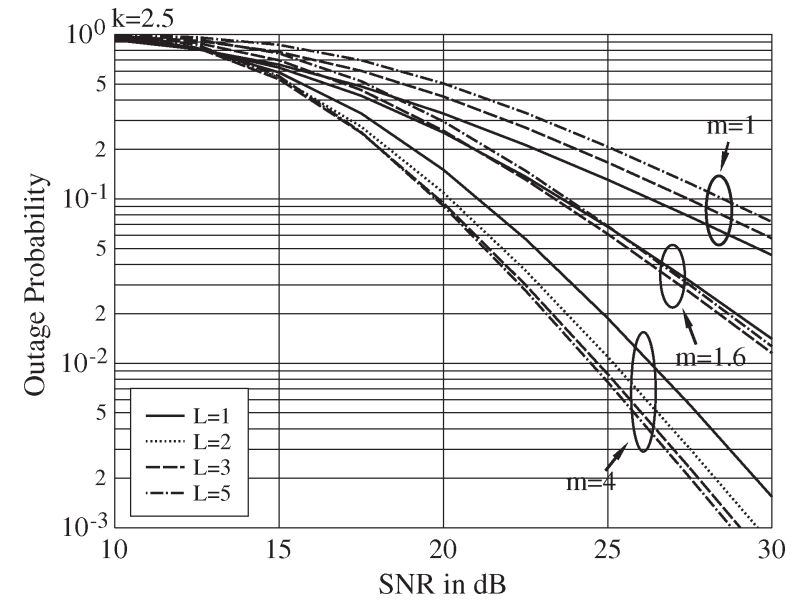

Fig. 7. Outage probability of one-, two-, three-, or five-hop links when communicating over $K_{G}$ fading channels with $\Omega_{l}=\Omega=(L / 5)^{3}$.

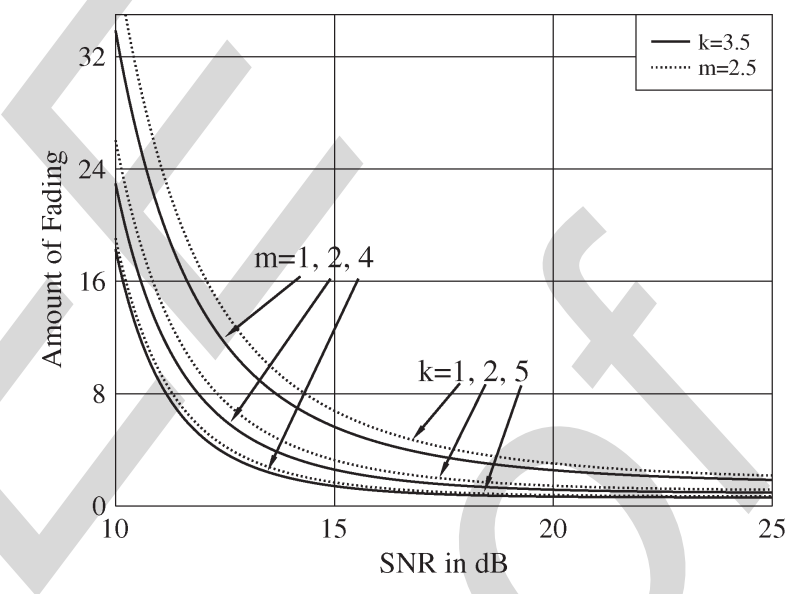

Fig. 8. Amount of fading of three-hop links when communicating over $K_{G}$ fading channels with $\Omega_{l}=\Omega=(3 / 5)^{3}$ and other various shaping parameters, as shown in the figure.

formance. Explicitly, when given the total propagation distance 405 $\left(L_{\max }=5\right)$, shadowing $(k=2.5)$, and the small-scale fading 406 of a communication link, there exists an optimum number of 407 hops, which yields the lowest BER or lowest outage proba- 408 bility. Specifically, when the small-scale fading is very severe 409 corresponding to $m=0.5$ in Fig. 6 and $m=1$ in Fig. 7, using 410 only one-hop link results in the best performance. When $m=1411$ in Fig. 6 and $m=1.6$ in Fig. 7, depending on the available 412 SNR, a three- or five-hop link may perform best. Finally, 413 when the channel quality in terms of the small-scale fading is 414 very good, for example, when $m=3$ in Fig. 6 and $m=4$ in 415 Fig. 7, generally, the link is beneficial to using as many hops as 416 possible to attain the best BER and outage performance.

The AOF of a three-hop $(L=3)$ link was numerically eval- 418 uated based on (9), which is shown in Fig. 8, when various 419 sets of parameters for the $K_{G}$ fading channels were considered. 420 Explicitly, given $k=3.5$, the fading becomes severer, as the 421 value of $m$ decreases. Similarly, given $m=2.5$, the fading 422 becomes more severe as the value of $k$ decreases.

In Fig. 9, we compare the end-to-end LCR of the fading over 424 a three-hop link when the communication channels over the 425 three hops are modeled as the i.i.d. $K_{G}$ fading channels with 426 various shaping parameters, as shown in the figure. In Fig. 9, the 427 


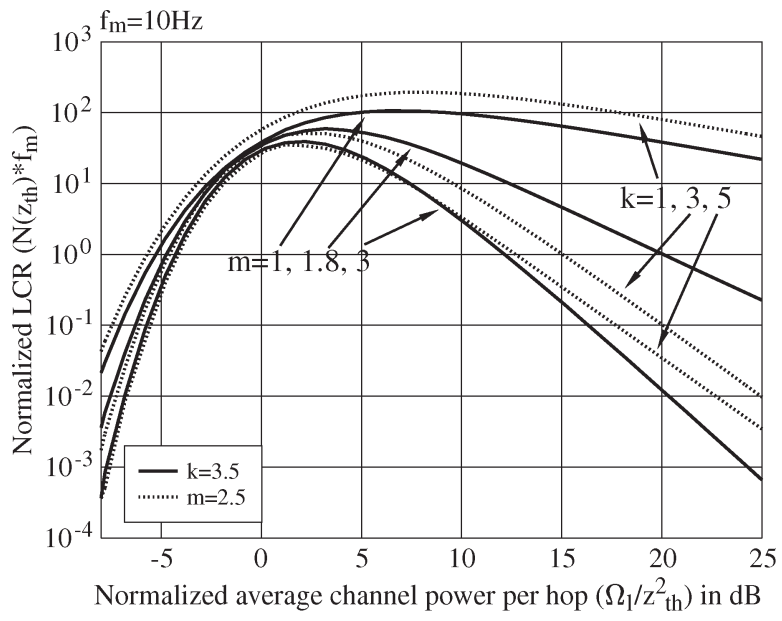

Fig. 9. Level-crossing rate of three-hop links when communicating over $K_{G}$ fading channels with $\Omega_{l}=\Omega=(3 / 5)^{3}$ and other various shaping parameters, as shown in the figure.

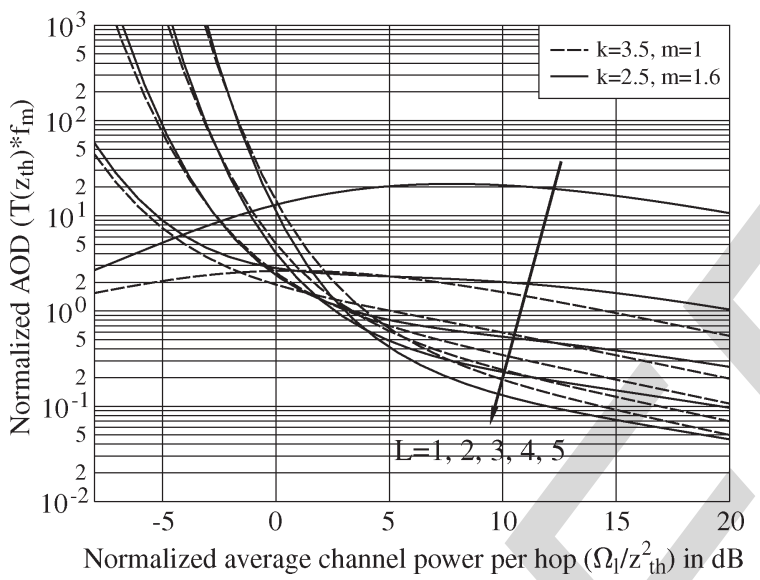

Fig. 10. AOD of multihop links when communicating over $K_{G}$ fading channels with $\Omega_{l}=\Omega=(3 / 5)^{3}$ and other various shaping parameters, as shown in the figure.

428 results were numerically evaluated based on (34) when given 429 the maximum Doppler frequency shift $f_{m}=10 \mathrm{~Hz}$ for any of 430 the three hops. From the results of Fig. 9, it seems that the 431 LCR of the three-hop link considered is sensitive more to the 432 change of the small-scale fading determined by parameter $m$ 433 than to the varying of the shadowing explained by parameter $k$. 434 When the average channel power per hop is low, the LCR is low 435 since, in this case, the three-hop channel stays mainly at the 436 "poor" state without many chances going to the "good" state. 437 Similarly, when the average channel power is high, the LCR is 438 also low. This is because, in this case, the three-hop channel 439 stays mainly at the "good" state without much fluctuation. By 440 contrast, as shown in Fig. 9, there is a range for the average 441 channel power, which results in the three-hop channel highly 442 fluctuating, yielding relatively high LCR. Finally, from Fig. 9, 443 we observe that, for a given value of the average channel power, 444 the three-hop channel becomes more fluctuated, as the value of $445 k$ or $m$ decreases.

446 Finally, Fig. 10 shows the normalized AOD of the links 447 having different numbers of hops when operated in $K_{G}$ fading 448 channels with the parameters as shown in the figure. Note that, 449 for Fig. 10, we assumed that all the hops had the same max- imum Doppler frequency shift expressed as $f_{m}$. In this case, 450 after being normalized by the maximum Doppler frequency 451 shift, the AOD is independent of $f_{m}$. As shown in Fig. 10, when 452 the average channel power is low, such as $\Omega_{l} / z_{\text {th }}^{2}<-5 \mathrm{~dB}, 453$ the AOD of the one-hop link may be significantly shorter 454 than the AOD of the multihop links. By contrast, when the 455 average channel power is high, such as $\Omega_{l} / z_{\mathrm{th}}^{2}>5 \mathrm{~dB}$, the 456 AOD decreases as the number of hops increases. The principles 457 behind the aforementioned observation may be explained as 458 follows: When the average channel power is low, each of the 459 hops is an unreliable channel. Hence, the multihop link has 460 a higher chance to stay at the "poor" state as the number of 461 hops increase. By contrast, when the average channel power 462 is high, due to the fluctuation of the component channels, 463 the multihop channel oscillates the server as the number of 464 hops increases. Correspondingly, the AOD of the multihop link 465 becomes shorter as the number of hops increases.

466

\section{CONCLUSION}

467

The SER, outage probability, and second-order statistics, 468 including both LCR and AOD, of multihop links have been in- 469 vestigated, when communicating over the $K_{G}$ fading channels, 470 which can simultaneously take into account the propagation 471 path loss, shadowing, and small-scale fading. Our studies and 472 performance results show that the $K_{G}$ channel model and the 473 expressions derived in this contribution are highly efficient 474 for evaluating the SER and outage performance of multihop 475 communication links, as well as for revealing the statistical 476 behavior of multihop channels. Given the distance of a trans- 477 mission link and the corresponding fading channel determined 478 by the parameters for the propagation path loss, shadowing, 479 and small-scale fading, our studies show that there exists an 480 optimum number of hops for signal delivery, which results in 481 the best SER and outage performance. In general, when the 482 channel is very poor, one-hop direct transmission is the desired 483 signal transmission option. When the quality of the channels 484 improves, the communication link expects more hops to aug- 485 ment the SER and outage performance. If the channels are very 486 reliable, it seems that the best SER and outage performance are 487 attained when the communication link uses as many hops as 488 possible.

\section{9}

\section{REFERENCES}

490

[1] T. S. Rappaport, Wireless Communications Principles and Practice, 491 2nd ed. New York: Prentice-Hall, 2002.

[2] M. K. Simon and M.-S. Alouini, Digital Communication Over Fading 493 Channels. New York: Wiley, 2005.

[3] A. Abdi and M. Kaveh, "On the utility of gamma PDF in modeling shadow 495 fading (slow fading)," in Proc. IEEE 49th Veh. Technol. Conf., Jul. 1999, 496 vol. 3, pp. 2308-2312.

[4] P. M. Shankar, "Error rates in generalized shadowed fading channels," 498 Wireless Pers. Commun., vol. 28, no. 3, pp. 233-238, Feb. 2004.

[5] P. Shankar, "Outage probabilities in shadowed fading channels using a 500 compound statistical model," Proc. Inst. Elect. Eng.-Commun., vol. 152, 501 no. 6, pp. 828-832, Dec. 2005.

[6] P. Bithas, N. Sagias, P. Mathiopoulos, G. Karagiannidis, and 503 A. Rontogiannis, "On the performance analysis of digital communications 504 over generalized- $k$ fading channels," IEEE Commun. Lett., vol. 10, no. 5, 505 pp. 353-355, May 2006.

[7] M. Hasna and M.-S. Alouini, "Outage probability of multihop transmis- 507 sion over Nakagami fading channels," IEEE Commun. Lett., vol. 7, no. 5, 508 pp. 216-218, May 2003. 
510 [8] L. Wu, J. Lin, K. Niu, and Z. He, "Performance of dual-hop transmissions 511 with fixed gain relays over generalized- $k$ fading channels," in Proc. IEEE $512 \quad$ ICC, Jun. 2009, pp. 1-5.

513 [9] L. Yang, M. Hasna, and M.-S. Alouini, "Average outage duration of 514 multihop communication systems with regenerative relays," IEEE Trans. 515 Wireless Commun., vol. 4, no. 4, pp. 1366-1371, Jul. 2005.

516 [10] A. Krantzik and D. Wolf, "Distribution of the fading-intervals of modified 517 Suzuki processes," in Signal Processing V: Theories and Applications, 518 L. Torres, E. Masgrau, and M. A. Lagunas, Eds. Amsterdam, The 519 Netherlands: Elsevier, 1990, pp. 361-364.

520 [11] N. C. Beaulieu and J. Hu, "A closed-form expression for the outage 521 probability of decode-and-forward relaying in dissimilar Rayleigh fading channels," IEEE Commun. Lett., vol. 10, no. 12, pp. 813-815, Dec. 2006.

12] I. Gradshteyn and I. Ryzhik, Table of Integrals, Series, and Products., 7th ed. Amsterdam, The Netherlands: Elsevier, 2007.

13] G. P. Efthymoglou, "On the performance analysis of digital modulations in generalized- $k$ fading channels," Wireless Pers. Commun., 2011. 10.1007/s11277-011-0277-8.

14] L.-L. Yang and H.-H. Chen, "Error probability of digital communications using relay diversity over Nakagami- $m$ fading channels," IEEE Trans. Wireless Commun., vol. 7, no. 5, pp. 1806-1811, May 2008.

15] The Wolfram Functions Site. [Online]. Available: http://functions. wolfram.com

16] I. Trigui, A. Laourine, S. Affes, and A. Stephenne, "On the level crossing rate and average fade duration of composite multipath/shadowing channels," in Proc. IEEE GLOBECOM, New Orleans, LA, Nov. 30-Dec. 4, 2008, pp. 1-5.

17] P. S. Bithas, P. T. Mathiopoulos, and S. A. Kotsopoulos, "Diversity reception over generalized- $K\left(K_{G}\right)$ fading channels," IEEE Trans. Wireless Commun., vol. 6, no. 12, pp. 4238-4243, Dec. 2007.

18] G. L. Stuber, Principles of Mobile Communication. Norwell, MA: Kluwer, 2000.

19] S. Rice, "Statistical properties of a sine wave plus random noise," Bell Syst. Tech. J., vol. 27, no. 1, pp. 109-157, Jan. 1948.

20] M. D. Yacoub, J. E. V. Bautista, and L. Guerra de Rezende Guedes, "On higher order statistics of the Nakagami-m distribution," IEEE Trans. Veh. Technol., vol. 48, no. 3, pp. 790-794, May 1999.

21] S. Primak and V. Kontorovich, "On the second order statistics of generalized Gamma process," in Proc. IEEE 17th Int. Symp. Pers., Indoor Mobile Radio Commun., Sep. 2006, pp. 1-5.

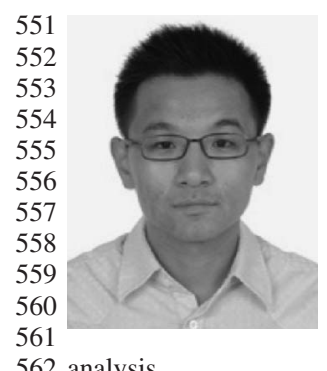

562 analysis.

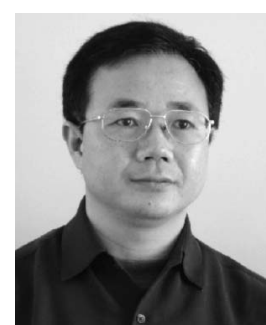

Lie-Liang Yang (M'98-SM'02) received the 563 B.Eng. degree in communications engineering from 564 Shanghai TieDao University, Shanghai, China, in 565 1988 and the M.Eng. and Ph.D. degrees in commu- 566 nications and electronics from Northern (Beijing) 567 Jiaotong University, Beijing, China, in 1991 and 568 1997, respectively.

From June 1997 to December 1997, he was a Vis- 570 iting Scientist with the Institute of Radio Engineering 571 and Electronics, Academy of Sciences of the Czech 572 Republic, Prague, Czech Republic. Since December 573 1997, he has been with the University of Southampton, Southampton, U.K., 574 where he is currently a Professor of wireless communications with the School of 575 Electronics and Computer Science. He has authored more than 270 research pa- 576 pers in journals and conference proceedings, authored/coauthored three books, 577 and authored several book chapters. He is currently an Associate Editor for the 578 Journal of Communications and Networks and the Security and Communication 579 Networks Journal. His research interests include wireless communications, 580 networking, and signal processing.

Dr. Yang is a Fellow of the Institution of Engineering and Technology. He is 582 currently an Associate Editor for the IEEE TRANSACTIONS ON VEHICULAR 583 TECHNOLOGY.

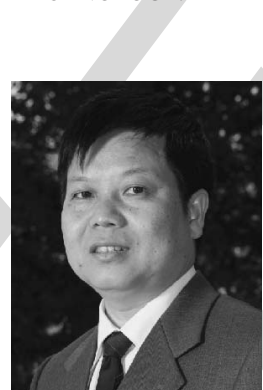

Zhangdui Zhong was born in May 1962.

$\mathrm{He}$ is currently the Professor and Director of 586 doctor degree candidates with Beijing Jiaotong Uni- 587 versity, Beijing, China. He is also the Director of 588 the Modern Telecommunications Research Institute, 589 Beijing Jiaotong University, and the Chief Scien- 590 tist of the Ministry Railways of China. He has au- 591 thored more than 90 scientific research papers. His 592 research interests include railway wireless network 593 systems, control theory and techniques for wireless 594 railway systems, and Global System for Mobile 595 Communications-Railway systems.
Jianfei Cao (S'10) received the B.Eng. degree from Hunan University, Changsha, China, in 2006. He is currently working toward the Dr.Eng. degree with the State Key Laboratory of Railway Traffic Control and Safety, Beijing Jiaotong University, Beijing, China. From September 2009 to October 2010, he did research as a Visiting Ph.D. student with the School of Electronics and Computer Science, University of Southampton, Southampton, U.K.

His research interests include cooperative communication, relaying techniques, and performance 


\section{AUTHOR QUERIES}

\section{AUTHOR PLEASE ANSWER ALL QUERIES}

AQ1 = Please provide volume, issue number, page range and publication date in Ref. [13].

AQ2 = Please provide educational background.

END OF ALL QUERIES 